\title{
Crystal Growth of the Lennard-Jones (100) Surface by Means of Equilibrium and Nonequilibrium Molecular Dynamics
}

\author{
W. J. Briels and H. L. Tepper \\ Chemical Physics Laboratory, University of Twente, P.O. Box 217, 7500 AE Enschede, The Netherlands
}

(Received 27 June 1997)

\begin{abstract}
On the basis of Onsager's hypothesis a new method is presented to calculate growth rate constants of various crystal faces from the fluctuations of interfaces during $N V T$ simulations. The method is applied to the (100) face of a Lennard-Jones crystal grown from the melt. The results are in perfect agreement with those obtained by means of NPT nonequilibrium simulations. The new method allows for much better statistics at the cost of much less computation time. The use of Onsager's hypothesis to derive the microscopic expression for the growth rate constant may serve as an example for applications in other fields. [S0031-9007(97)04878-3]
\end{abstract}

PACS numbers: 81.10.Aj, 05.20.Dd, 64.70.Dv, 71.15.Pd

The study of growth rates of crystals from their melt, or from supersaturated solutions, besides being an interesting theoretical problem, is of importance for the prediction of macroscopic growth morphologies of crystals. The rheological properties of particulate systems, be it liquids in which small crystallites are suspended or just granular materials consisting of small crystallites, very much depend on the morphologies of the crystallites.

Since the crystal-melt interface, being a combination of two dense phases, is not easily accessible to experimental measurements, molecular dynamics simulations can be of great help in the study of these systems. Several studies of the crystal-melt interface of Lennard-Jones systems, consisting of atoms which interact by pairwise potentials of the form

$$
\Phi_{\mathrm{LJ}}(r)=4 \epsilon\left[\left(\frac{\sigma}{r}\right)^{12}-\left(\frac{\sigma}{r}\right)^{6}\right]
$$

have been reported in the literature, mainly by Broughton etal. [1-5]. Among other things, they performed nonequilibrium molecular dynamics simulations to calculate the growth rates of various crystal faces over a wide range of temperatures below the melting point. In this paper, we present a method to calculate growth rates from fluctuations of the interface during an equilibrium simulation. We have applied this method to study the growth rate of the (100) face of a Lennard-Jones crystal growing from its melt, and checked the results by performing nonequilibrium simulations for very small deviations from equilibrium.

In order to be able to decide whether a particle belongs to the crystal or to the melt, we have constructed a very simple recognition function. What distinguishes a particle in the crystal from a particle in the liquid is the fact that its surroundings have octahedral symmetry. We therefore have constructed, using the methods of Ref. [6], a function $X(\mathbf{r})$, which for every vector $\mathbf{r}$ pointing from the particle under investigation to one of its nearest neighbors, in a perfect crystal, takes the same value. When the fourfold symmetry axes of the octahedron are chosen along the three Cartesian axes, the simplest such function reads

$$
X(\mathbf{r})=x^{4} / r^{4}+y^{4} / r^{4}+z^{4} / r^{4}-3 / 5 .
$$

The order parameter $\Psi$, which discriminates between crystal and liquid particles was next defined by

$$
\Psi=\sqrt{\frac{\left\langle X^{2}\right\rangle-\langle X\rangle^{2}}{\langle X\rangle^{2}}}
$$

where the averages were taken over all nearest neighbors residing within a cutoff radius of $1.39 \sigma$. A slight amelioration was obtained by referring the positions of the neighbors, not to the central particle, but to the center of mass of the neighbors. The distributions of the values of $\Psi$ during a simulation of a pure crystal and a simulation of a pure liquid are plotted in Fig. 1. It is seen that by calling crystal particles those particles for which $\Psi<0.5$ and liquid particles those for which $\Psi>0.5$, a perfect discriminator is defined. It is important to realize that crystalline structure will partly persist in the liquid phase near the interface. As a result our discriminator will slightly overestimate the number of solid particles. We expect, however, that changes in the number of solid particles will be correctly monitored.

In the case of simple monoatomic systems the melting temperature is found to be above the roughening transition. Growth occurs at any point on the surface, and the growth mode is called normal growth [7-9]. In the case of small deviations from equilibrium, the growth rate of such a surface is proportional to the difference of the chemical potentials of the liquid and the solid phases, i.e.,

$$
R=k d \frac{\mu_{l}-\mu_{s}}{k_{B} T}
$$

where $d$ is the interplanar distance parallel to the interface. The growth rate is related to the increase of the number of solid particles $d N_{s} / d t$ by

$$
R=\frac{d}{A / a} \frac{d N_{s}}{d t},
$$




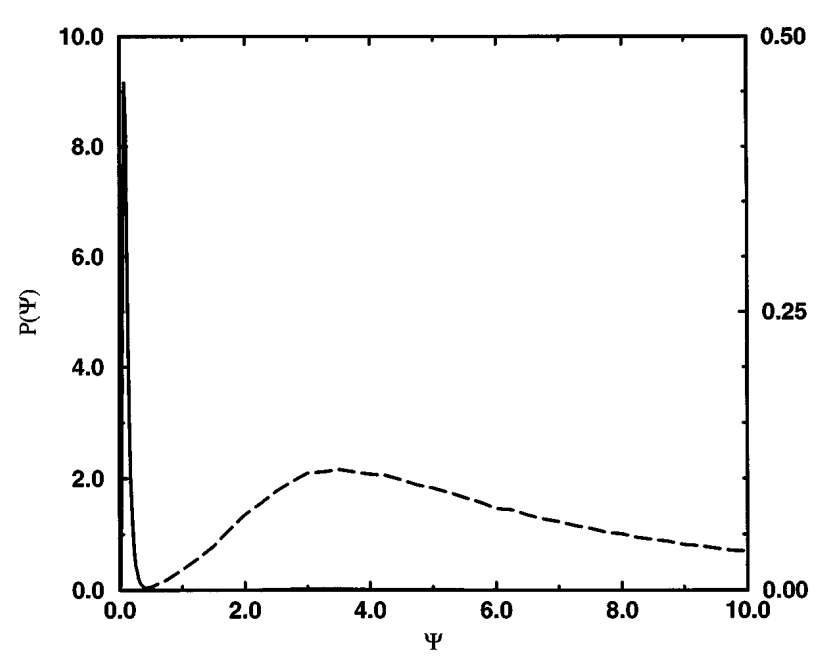

FIG. 1. Distribution of the recognition function $\Psi$ in bulk solid (solid line, left scale) and in bulk liquid (dashed line, right scale).

with $a$ the specific area taken by a solid particle, and $A$ the total area of the surface.

When the system is in equilibrium, no net growth takes place, and on average $R$ equals zero. Instantaneous fluctuations of the number of solid particles do occur, however, and their dynamics is related to the above equations by means of Onsager's hypothesis. This hypothesis states $[10,11]$ that slow equilibrium fluctuations on average decay according to macroscopic laws. We now first derive the macroscopic laws governing the relaxation of a small deviation $\Delta N_{s}=N_{s}-N_{s}^{\text {eq }}$ of the number of solid particles from its equilibrium value $N_{s}^{\mathrm{eq}}$, in a closed system with constant volume and constant temperature. First we relate the difference between the chemical potentials occurring in Eq. (4) to the deviations from equilibrium of the molar volumes of both phases:

$$
\mu_{l}-\mu_{s}=-\frac{1}{\kappa_{l}} \Delta v_{l}+\frac{1}{\kappa_{s}} \Delta v_{s}
$$

where $\kappa$ is the isothermal compressibility. In order to calculate $\Delta v_{l}$ and $\Delta v_{s}$ we use the following relations:

$$
\begin{gathered}
\left(N_{l}^{\mathrm{eq}}-\Delta N_{s}\right)\left(\boldsymbol{v}_{l}^{\mathrm{eq}}+\Delta \boldsymbol{v}_{l}\right)+ \\
\left(N_{s}^{\mathrm{eq}}+\Delta N_{s}\right)\left(v_{s}^{\mathrm{eq}}+\Delta \boldsymbol{v}_{s}\right)=V, \\
\frac{\Delta \boldsymbol{v}_{l}}{\boldsymbol{v}_{l}^{\mathrm{eq}} \kappa_{l}}=-\Delta P=\frac{\Delta \boldsymbol{v}_{s}}{\boldsymbol{v}_{s}^{\mathrm{eq}} \kappa_{s}},
\end{gathered}
$$

where $N_{l}^{\mathrm{eq}}=N-N_{s}^{\mathrm{eq}}$. The second of these equations expresses uniformity of pressure during the simulations. This means that we assume that pressure gradients relax much faster than the time scales we are interested in. Solving Eqs. (7) and (8) for $\Delta v_{l}$ and $\Delta v_{s}$ to first order in $\Delta N_{s}$, and using the result in Eqs. (4)-(6), we obtain

$$
\begin{gathered}
\frac{d \Delta N_{s}}{d t}=-\frac{1}{\tau} \Delta N_{s}, \\
\frac{1}{\tau}=k \frac{A / a}{N}\left(\frac{\left(v_{l}^{\mathrm{eq}}-v_{s}^{\mathrm{eq}}\right)^{2}}{v_{s}^{\mathrm{eq}} \kappa_{s} f_{s}^{\mathrm{eq}}+v_{l}^{\mathrm{eq}} \kappa_{l} f_{l}^{\mathrm{eq}}}\right) \frac{1}{k_{B} T},
\end{gathered}
$$

where $f_{l}^{\mathrm{eq}}=N_{l}^{\mathrm{eq}} / N$ and $f_{s}^{\mathrm{eq}}=N_{s}^{\mathrm{eq}} / N$. Equation (9) says that $\Delta N_{s}$ decays from its initial value according to a simple exponential law.

We are now in the position to make use of Onsager's hypothesis in the form $\left\langle\Delta N_{s}(t)\right\rangle_{\Delta N_{s}(0)}=$ $\Delta N_{s}(0) \exp (-t / \tau)$, from which we find

$$
C(t)=\frac{\left\langle\Delta N_{s}(t) \Delta N_{s}(0)\right\rangle}{\left\langle\Delta N_{s}(0)^{2}\right\rangle}=\exp \{-t / \tau\}
$$

All simulations were done with the GROMOS package [12]. First, separate $N P T$ simulations with $P=2.546 \times$ $10^{-3} \epsilon / \sigma^{3}$ and $T=0.646 \epsilon / k_{B}$ were carried out for a crystal and a liquid. The rates with which velocities and box volume were rescaled [13], were controlled by the relaxation times $\tau_{T}=0.0747\left(m \sigma^{2} / \epsilon\right)^{1 / 2}$ and $\tau_{P} / \kappa=$ $222.6(m \epsilon)^{1 / 2} / \sigma^{2}$, with $m$ the particle's mass. The time step of the numerical integration of Newton's equations was $\Delta t=0.0015\left(m \sigma^{2} / \epsilon\right)^{1 / 2}$. The temperature was close enough to the equilibrium temperature for no structural changes to occur during either of the two simulations. Next, the crystal box was surrounded by two liquid boxes along the $y$ axis. Subsequent simulations were done in the $N V T$ ensemble with $T=0.646 \epsilon / k_{B}$; the number of particles was 1378 , and the box dimensions were $L_{x}=L_{z}=8.069 \sigma$ and $L_{y}=24.206 \sigma$. Note that two interfaces occur in the box and that $A$ in Eqs. (5) and (10) denotes the total surface area.

Correlation function $C(t)$ from Eq. (11) is plotted in Fig. 2. After a short transient time of about $1.5\left(m \sigma^{2} / \epsilon\right)^{1 / 2}$, this correlation function decays exponentially with time constant $\tau=9.887\left(m \sigma^{2} / \epsilon\right)^{1 / 2}$, which amounts to $k=0.5269\left(m \sigma^{2} / \epsilon\right)^{-1 / 2}$. The value of $k$ was calculated from Eq. (10), using $d=0.8113 \sigma, v_{s}^{\mathrm{eq}}=$ $1.0564 \sigma^{3}, v_{l}^{\mathrm{eq}}=1.200 \sigma^{3}, \quad \kappa_{s}=0.00428 \sigma^{3} / \epsilon,{ }_{\mathrm{eq}} \kappa_{l}=$ $0.01412 \sigma^{3} / \epsilon, N_{s}^{\mathrm{eq}}=540.7$, and $N_{l}^{\mathrm{eq}}=837.3$. $v_{s}^{\mathrm{eq}}, v_{l}^{\mathrm{eq}}$, and $d$ were measured from the bulk parts of the

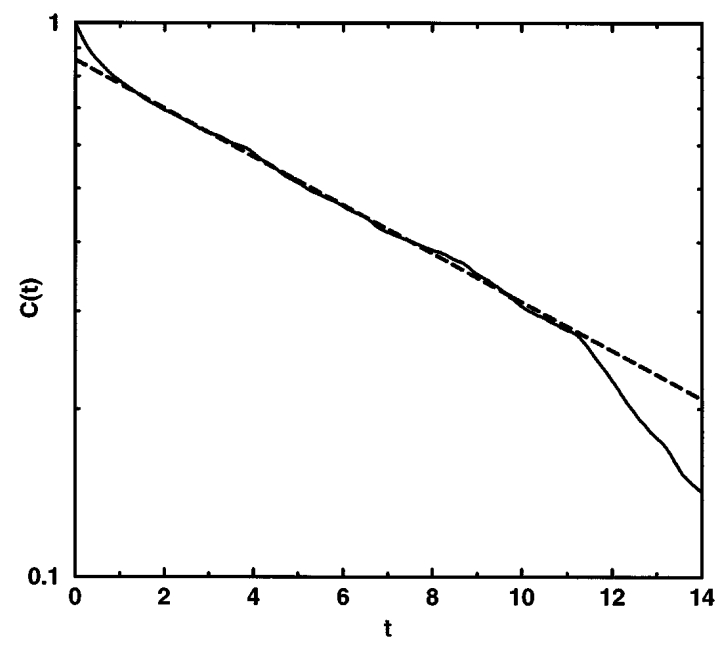

FIG. 2. Normalized time dependent auto correlation function of $\Delta N_{s}$. Time scale in units of $\left(m \sigma^{2} / \epsilon\right)^{1 / 2}$. 


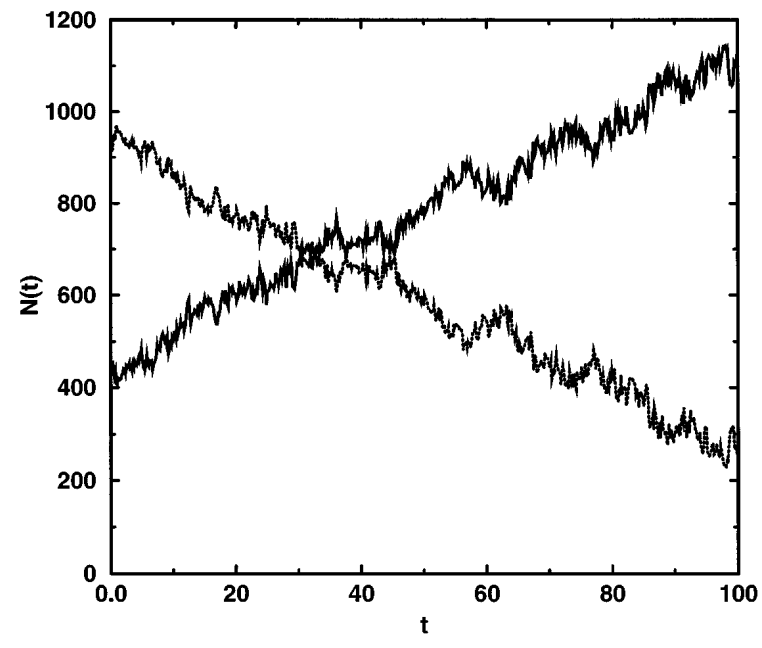

FIG. 3. Characteristics of a growing crystal. The solid line denotes the number of solid particles and the dashed line the number of liquid particles. Time scale in reduced units. $T=0.612 \epsilon / k_{B}$.

respective phases in the equilibrium simulation; $\kappa_{l}$ was calculated using the equation of state given by Nicholas et al. [14]; $\kappa_{s}$ was calculated according to $\kappa_{s} / \kappa_{l}=\Delta v_{s} / \Delta v_{l} \times v_{l} / v_{s}$ with $v_{s}$ and $v_{l}$ the specific volumes of the initial solid and liquid boxes, and $\Delta v_{s}=v_{s}^{\mathrm{eq}}-v_{s}$ and $\Delta v_{l}=v_{l}^{\mathrm{eq}}-v_{l} ; N_{s}^{\mathrm{eq}}$ and $N_{l}^{\mathrm{eq}}$ were calculated from $N_{s}^{\mathrm{eq}} \boldsymbol{v}_{s}^{\text {eq }}+N_{l}^{\mathrm{eq}} \boldsymbol{v}_{l}^{\mathrm{eq}}=V$ and $N_{s}^{\mathrm{eq}}+N_{l}^{\mathrm{eq}}=N$.

It is seen that for times larger than about 11 $\left(m \sigma^{2} / \epsilon\right)^{1 / 2}$, large deviations from exponential decay occur. These deviations reflect the influence of long-time temperature oscillations, caused by insufficient removal and supply of latent heat of crystallization and melting, respectively.

In order to check our results, we have done nonequilibrium simulations in the $N P T$ ensemble with $P=2.546 \times$ $10^{-3} \epsilon / \sigma$ at various temperatures around the equilibrium temperature. An example of the evolution of $N_{s}(t)$ with time during such a simulation is given in Fig. 3. The fact that $N_{s}$ is a linear function of time means that we have very well succeeded in keeping constant the nonequilibrium conditions. Applying parameters for argon, the growth rate amounts to $14 \mathrm{~m} / \mathrm{s}$ in good agreement with the results of Burke and Broughton.

For small deviations from equilibrium we may write

$$
R=-k d \frac{h_{l}^{\mathrm{eq}}-h_{s}^{\mathrm{eq}}}{k_{B} T^{\mathrm{eq}}} \frac{\Delta T}{T^{\mathrm{eq}}},
$$

i.e., the growth rate is proportional to $\Delta T=T-T^{\mathrm{eq}}$. In Fig. 4, the growth rates obtained for various temperatures are collected. The straight line is obtained from Eq. (12) using $k$ from the equilibrium simulation and $h_{l}^{\mathrm{eq}}-h_{s}^{\mathrm{eq}}=$ $1.01 \epsilon . h_{l}^{\mathrm{eq}}$ and $h_{s}^{\mathrm{eq}}$ were obtained from short $N V T$ simulations at the respective equilibrium densities and $T=0.646 \epsilon / k_{B}$. It is seen that this line very well

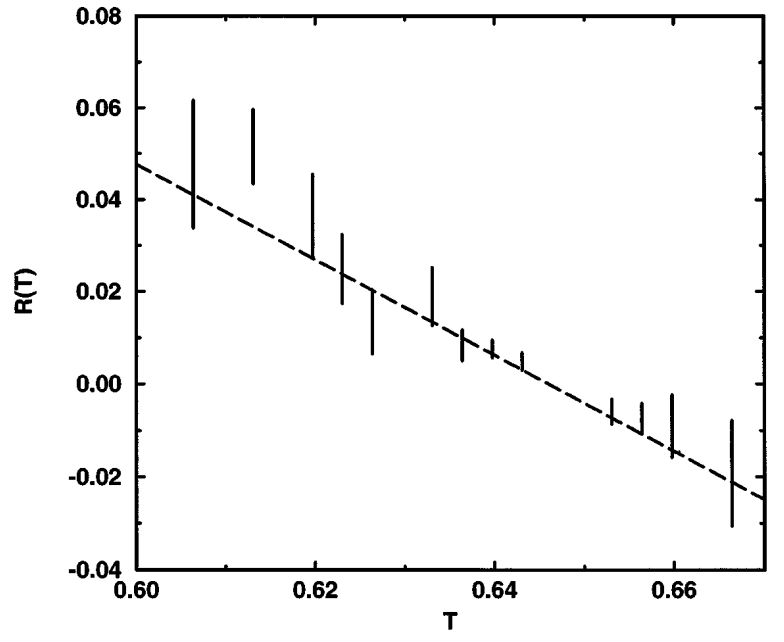

FIG. 4. Nonequilibrium results of the growth rate vs temperature (both in reduced units). The straight line represents growth rates calculated with the growth rate constant $k$ obtained with the equilibrium simulation.

represents the data, and that therefore the equilibrium and nonequilibrium simulations are in good agreement with each other.

The error bars in Fig. 4 roughly indicate the maximum and minimum values of the corresponding growth rates. For large values of $|\Delta T|$ the system rapidly melts or crystallizes. As a result the runs are very short and the difference between maximum and minimum slopes are relatively large. One way to obtain better statistics is to repeat the same simulation several times. A second way might be to use much larger boxes. This, however, is not a realistic possibility because with such large growth or melting rates it will become increasingly difficult to maintain constant nonequilibrium conditions. As a consequence, the nonequilibrium method which asks for many simulations for many different values of $\Delta T$ is much more costly than one equilibrium simulation. One disadvantage of the equilibrium method is that the (finite size) equilibrium temperature must be known fairly accurately, in order for the pressure not to change too much during the initial part of the $N V T$ run. As a final remark we notice that the relaxation time which is actually measured, i.e., the relaxation time $\tau$, is proportional to $N / A$, i.e., to the box size normal to the interface. This size must therefore not take part in the thermodynamic limit.

[1] J. Q. Broughton, G. H. Gilmer, and K. A. Jackson, Phys. Rev. Lett. 49, 1496 (1982).

[2] J. Q. Broughton and G. H. Gilmer, J. Chem. Phys. 79, 5095 (1983).

[3] J. Q. Broughton and G. H. Gilmer, J. Chem. Phys. 84, 5749 (1986). 
[4] J. Q. Broughton and G. H. Gilmer, J. Chem. Phys. 84, 5759 (1986).

[5] E. Burke, J. Q. Broughton, and G. H. Gilmer, J. Chem. Phys. 89, 1030 (1988).

[6] W. J. Briels, J. Chem. Phys. 73, 1850 (1980).

[7] P. Bennema and J.P. van der Eerden in Morphology of Crystals, edited by I. Sunagawa (Terrapub, Tokyo, 1987), Chap. 1.

[8] B. Chalmers, Principles of Solidification (John Wiley \& Sons, New York, 1964), p. 44.

[9] I. V. Markov Crystal Growth for Beginners (World Scientific, New York, 1995).
[10] D. Chandler, Introduction to Modern Statistical Mechanics (Oxford University Press, Oxford, 1987).

[11] W. J. Briels, Theory of Simple Liquids (Uppsala University, Uppsala, 1993) [lecture notes].

[12] H. J.C. Berendsen and W.F. van Gunsteren, GROMOS Reference Manual (University of Groningen, Groningen, 1987).

[13] H. J. C. Berendsen, J. P. M. Postma, W.F. van Gunsteren, A. DiNola, and R. J. Haak, J. Chem. Phys. 81, 3684 (1984).

[14] J. J. Nicholas, K. E. Gubbins, W. B. Street, and D. J. Tildesley, Mol. Phys. 37, 1429 (1979). 